INPLASY

PROTOCOL

To cite: Pallavicini et al.

Commercial Off-The-Shelf

Video Games for Reducing

Stress and Anxiety: A PRISMA

Systematic Review. Inplasy

protocol 202130081. doi:

10.37766/inplasy2021.3.0081

Received: 23 March 2021

Published: 23 March 2021

Corresponding author:

Federica Pallavicini

federica.pallavicini@gmail.com

Author Affiliation:

Università degli Studi di

Milano-Bicocca

Support: None.

Review Stage at time of this

submission: Data extraction.

Conflicts of interest:

None declared.

\section{Commercial Off-The-Shelf Video Games for Reducing Stress and Anxiety: A PRISMA Systematic Review}

\author{
Pallavicini, F1; Pepe, A2; Mantovani, F³.
}

Review question / Objective: Commercial off-the-shelf (COTS) video games have revealed potential application for mental health, including the reduction of stress and anxiety. Using COTS video games rather than video games created ad hoc could have several advantages for the treatment of stress and anxiety, as well as other mental conditions, including their low-cost and ready-to-use, advanced graphic quality, and the possibility to reach millions of players worldwide. However, it is important to emphasize not all COTS video games are equal, and their effects strongly depend on specific characteristics of the game itself, such as its genre. As reported by a recent systematic review, among the genres that are effective in decreasing stress anxiety are the casual video games (CVGs), characterized by low cognitive loads and generally short time demands, such as Tetris or Angry Birds. Despite this, in addition to CVGs, there are also other genres of COTS video games that look particularly promising for decreasing stress and anxiety in individuals, such as exergames or survival horror games. Therefore, this systematic review aimed to describe the literature on the use of COTS video games for diminishing stress and anxiety, organizing the research along with critical variables.

INPLASY registration number: This protocol was registered with the International Platform of Registered Systematic Review and Meta-Analysis Protocols (INPLASY) on 23 March 2021 and was last updated on 12 August 2021 (registration number INPLASY202130081).

\section{INTRODUCTION}

Review question / Objective: Commercial off-the-shelf (COTS) video games have revealed potential application for mental health, including the reduction of stress and anxiety. Using COTS video games rather than video games created ad hoc could have several advantages for the treatment of stress and anxiety, as well as other mental conditions, including their low-cost and ready-to-use, advanced 
graphic quality, and the possibility to reach millions of players worldwide. However, it is important to emphasize not all COTS video games are equal, and their effects strongly depend on specific characteristics of the game itself, such as its genre. As reported by a recent systematic review, among the genres that are effective in decreasing stress anxiety are the casual video games (CVGs), characterized by low cognitive loads and generally short time demands, such as Tetris or Angry Birds. Despite this, in addition to CVGs, there are also other genres of COTS video games that look particularly promising for decreasing stress and anxiety in individuals, such as exergames or survival horror games. Therefore, this systematic review aimed to describe the literature on the use of COTS video games for diminishing stress and anxiety, organizing the research along with critical variables.

Condition being studied: In the last decades, several studies have reported that video games may be of benefit for reducing stress and anxiety. Computer games offer various positive emotionstriggering situations. Their fundamental objective is to entertain the player and elicit positive emotions (e.g., happiness). Still, as also reported by many players, video games offer the opportunity to get distracted and take a break from problems or negative thoughts, with positive effects on the player's emotional state. Interestingly, in addition to custom-made video games, also commercial off-the-shelf (COTS) video games have revealed potential applications for the reduction of stress and anxiety. Using COTS video games rather than video games created ad hoc could have several advantages for the treatment of stress and anxiety, as well as other mental conditions, including their low-cost and ready-to-use, advanced graphic quality, and the possibility to reach millions of players worldwide. However, it is important to emphasize not all COTS video games are equal, and their effects strongly depend on specific characteristics of the game itself, such as its genre. Therefore, this systematic review aimed to describe the literature on the use of COTS video games for diminishing stress and anxiety, organizing the research along with critical variables.

\section{METHODS}

Search strategy: Search Strategy: The search string will be: [("video game*") OR ("computer game*")] AND [("stress") OR ("anxiety") OR ("relaxation")] AND [("study") OR ("trial") OR ("training")].

Participant or population: All human participants (clinical and non-clinical population) included in studies evaluating COTS video games effects.

Intervention: COTS video games intervention.

Comparator: usual care intervention, nonvideo game group, or none.

Study designs to be included: Randomized controlled trial (i.e., a study design that randomly assigns participants into an experimental group or a control group), quasi-experimental (i.e., nonequivalent groups, pretest-posttest, and interrupted time series) or cross-sectional/ correlational (i.e., employing questionnaires and large samples) study.

Eligibility criteria: In line with the PRISMA guidelines, the authors (FP, AP, FM) will establish clear inclusion criteria to determine papers' eligibility for inclusion in the review. Only studies meeting the following criteria will be considered eligible for inclusion: - Types of participants: all human participants (clinical and nonclinical population); - Interventions: COTS video games played on a console, mobile console, PC, smartphone/tablet, or virtual reality devices; - Comparators: usual care intervention, non-video game group, or none; - Outcomes: levels of stress, anxiety, or both; - Study design: randomized controlled trial (i.e., a study design that randomly assigns participants into an experimental group or a control group), quasi-experimental (i.e., nonequivalent groups, pretest-posttest, and interrupted time series), or cross-sectional/ 
correlational (i.e., employing questionnaires and large samples) study. Papers published in English in peer-reviewed journals will be selected and subjected to the inclusion criteria as outlined above. According to PRISMA guidelines, the authors (FP, AP, FM) established a specific date range. Studies published between January 2006 and March 2021 will be selected. This date frame was chosen as papers first studying video games' effect on stress, and anxiety reduction appeared around the 2010s. Studies will be excluded if: (1) did not focus on the use of COTS games for diminishing stress and/or anxiety; (2) focused on games that did not meet the definition of COTS, that is, "games that one can purchase on the high street" [19], or rather purchasable in online or physical stores; (3) used a modified version in its mechanics or features of a COTS, that change a fundamental aspect of the game; (4) used custom-made games (i.e., serious games); (5) did not specify the title of the game used; (6) did not specify the average age or age range of the participants.

Information sources: Databases used in the search will be PsycINFO, Web of Science, Medline, IEEExplore, and the Cochrane Library. Additional articles will be identified via hand-searching and reviewing the reference lists of relevant papers.

Main outcome(s): Anxiety and/or stress levels. To assess the extent to which COTS video games elicit reductions in stress and/ or anxiety levels, papers will be studied for comparisons between measures of stress and/or anxiety levels at pre-versus postintervention.

Quality assessment / Risk of bias analysis: Study quality will be assessed by two researchers (FP and AP) using using the mixed methods appraisal tool. The mixed methods appraisal tool is designed for systematic reviews, including a combination of quantitative, qualitative, and mixed methods studies, and has been noted for its reliability and efficiency as a quality assessment protocol, and capability to concomitantly appraise methodological quality across a variety of empirical research. In line with PRISMA guidelines, an interrater process will be adopted and the degree of agreement will be assessed, to reduce risk of bias.

Strategy of data synthesis: Papers meeting inclusion criteria will be identified through database searches. After papers published in languages other than English, and duplicate instances of papers will be removed, remaining papers will be assessed using the inclusion and exclusion criteria outlined above. Initially, abstracts will be searched to assess a paper's eligibility for inclusion. If abstract information alone will be not sufficient to determine whether a paper met the criteria, the entire paper will be studied. The following data will be extracted: - Study characteristics: the sample included in the study (participants; mean age or age range); the research design used, categorized as a randomized controlled trial (i.e., a study design that randomly assigns participants into an experimental group or a control group), quasiexperimental (i.e., nonequivalent groups, pretest-posttest, and interrupted time series) or cross-sectional/correlational (i.e., employing questionnaires and large samples); the measures used for the assessment of outcomes (e.g., self-report questionnaires, physiological data, cognitive task); study outcomes (i.e., stress, anxiety or both and differences in the outcome measures related with playing COTS). - Videogames interventions characteristics: the game genre, categorized as CVGs, action video games; adventure video games; racing video games; sports video games, role-playing (RPG) video games, strategy video games, simulation video games, exergames; augmented reality (AR) games; the platform for the game (console, mobile console, PC, smartphone/tablet, virtual reality); time spent playing (duration and the total amount of sessions). These data will be extracted independently by two researchers (FP and AP) with disagreements resolved by a consensus between both researches (FP and AP).

Subgroup analysis: None. 
Sensitivity analysis: None.

Language: English.

Country(ies) involved: Italia.

Keywords: Stress, anxiety, video games, commercial off-the-shelf video games, mental health.

Contributions of each author:

Author 1 - Federica Pallavicini.

Email: federica.pallavicini@gmail.com

Author 2 - Alessandro Pepe.

Email: alessandro.pepe1@unimib.it

Author 3 - Fabrizia Mantovani.

Email: fabrizia.mantovani@unimib.it 